\title{
Microsatellite genotyping of Candida parapsilosis from Iranian clinical isolates
}

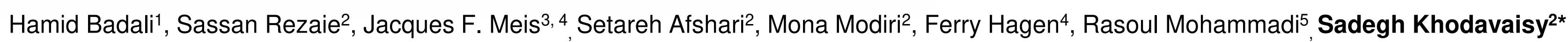
${ }^{1}$ Department of Medical Mycology and Parasitology/Invasive fungi Research Center, Mazandaran University of Medical Sciences, Sari, Iran; ${ }^{2 D}$ Department of Medical Mycology and Parasitology, Tehran University of Medical Sciences, Tehran, Iran; ${ }^{3}$ Department of Medical Microbiology and Infectious Diseases, Canisius- Wilhelmina Hospital, Nijmegen, The Netherlands; ${ }^{4}$ Centre of Expertise in Mycology Radboudumc/CWZ, Nijmegen, The Netherlands; Department of Medical Microbiology Nijmegen, The Netherlands, Radboudumc; 5Department of Medical Parasitology and Mycology, School of Medicine, Infectious Disease and Tropical Medicine Research Center, Isfahan University of Medical Sciences, Isfahan, Iran

Candida parapsilosis is one of the most frequent non-albicans Candida (NAC) species, which causes a broad spectrum of infections from superficial to invasive candidiasis. These species can lead to nosocomial infection, particularly in hospitalized neonatal and pediatric patients through catheters, intravascular devices, and hand carriage of the health care workers. Based on genotypic analysis, these outbreaks were probably due to crossinfections by health care workers. Among molecular methods, microsatellite analysis has been used for molecular typing of the species belonging to $C$. parapsilosis and other fungi. Therefore, the current study aimed to apply microsatellite analysis for genotyping of clinical C. parapsilosis isolates from Iran.

A total of 81 C. parapsilosis clinical isolates were investigated using molecular typing method. All clinical isolates were grown for $48 \mathrm{~h}$ on Sabouraud glucose agar (Merck, Germany) at $30^{\circ} \mathrm{C}$. DNA was extracted using the CinnaPure DNA isolation kit in accordance with the manufacturer's recommendations. The identities of $C$. parapsilosis isolates were confirmed by the partial sequencing of the internal transcribed spacer DNA region.

A panel of six short tandem repeat (STR) markers was used for typing all clinical isolates of $C$. parapsilosis. Generally, three trinucleotide repeat markers and three hexanucleotide markers were amplified in a multiplex polymerase chain reaction (PCR). In addition, each amplification primers were labelled at the $5^{\prime}$ side with 6-carboxy-fluorescein (6-FAM), either 6-carboxytetramethylrhodamine (TAMRA) or hexachloro-fluorescein (HEX). The similarities between the genotypes were visualized by constructing a minimum spanning tree (MST) using BioNumerics, version 6.0 (Applied Maths, St-Martens-Latem, Belgium). The discriminatory power was calculated by Simpson's index of diversity.

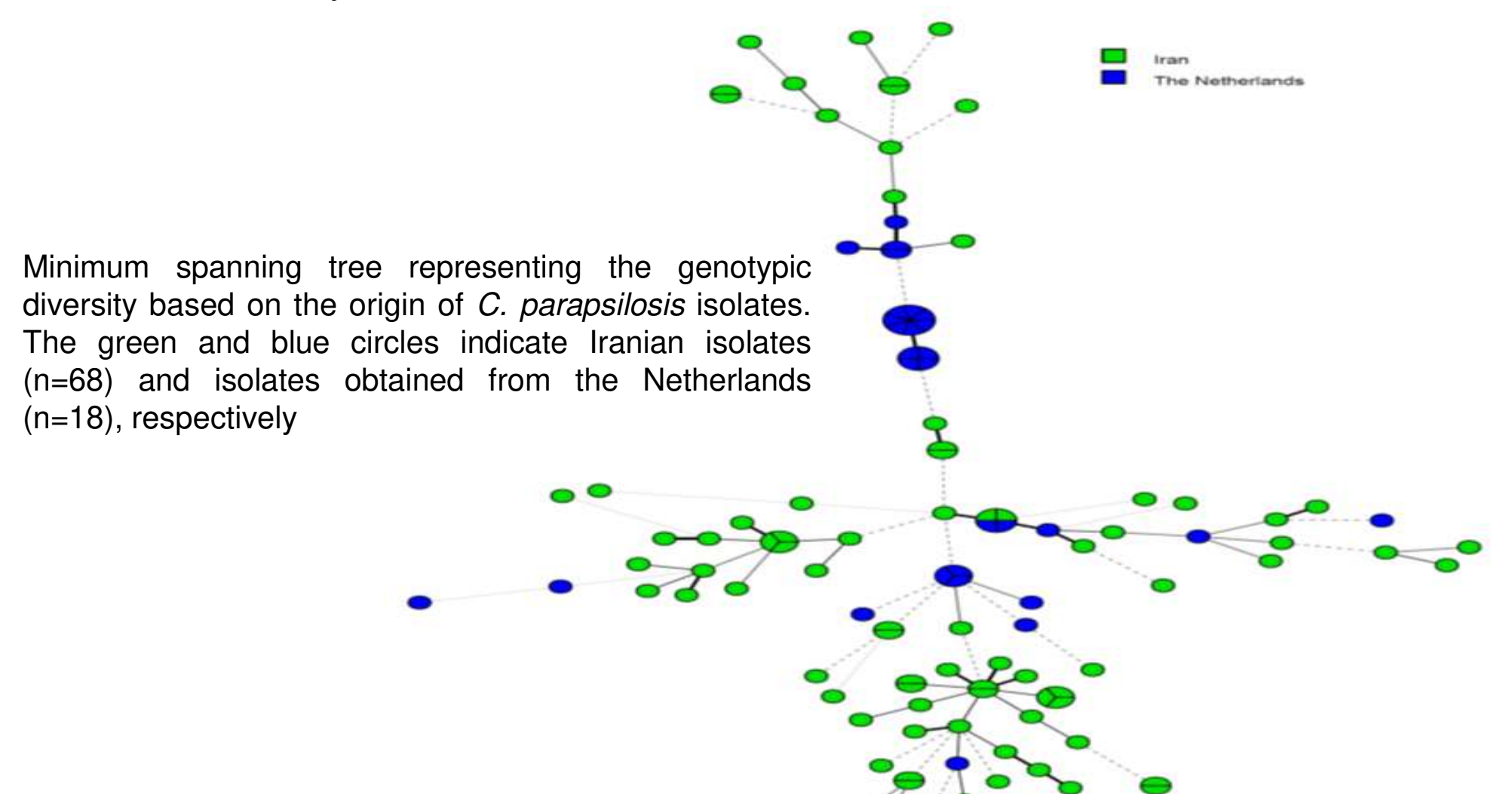

Totally, 81 clinical C. parapsilosis isolates were identified from a variety of clinical specimens, including nail scraping $(n=59)$, interdigital skin $(n=8)$, groin $(n=6)$, vaginal $(n=2)$, ear discharge $(n=2)$, skin $(n=2)$, hand $(n=1)$, and sputum $(n=1)$. The STR typing of 81 C. parapsilosis isolates demonstrated 68 separate genotypes. The genetic relatedness of clinical $C$. parapsilosis isolates is depicted in figure 1. Out of all genotypes, 57 cases were observed once, and the remaining 11 genotypes were identified for multiple times. Nine genotype clusters were shared between two C. parapsilosis isolates, and also two genotypes were shared among three $C$. parapsilosis isolates.

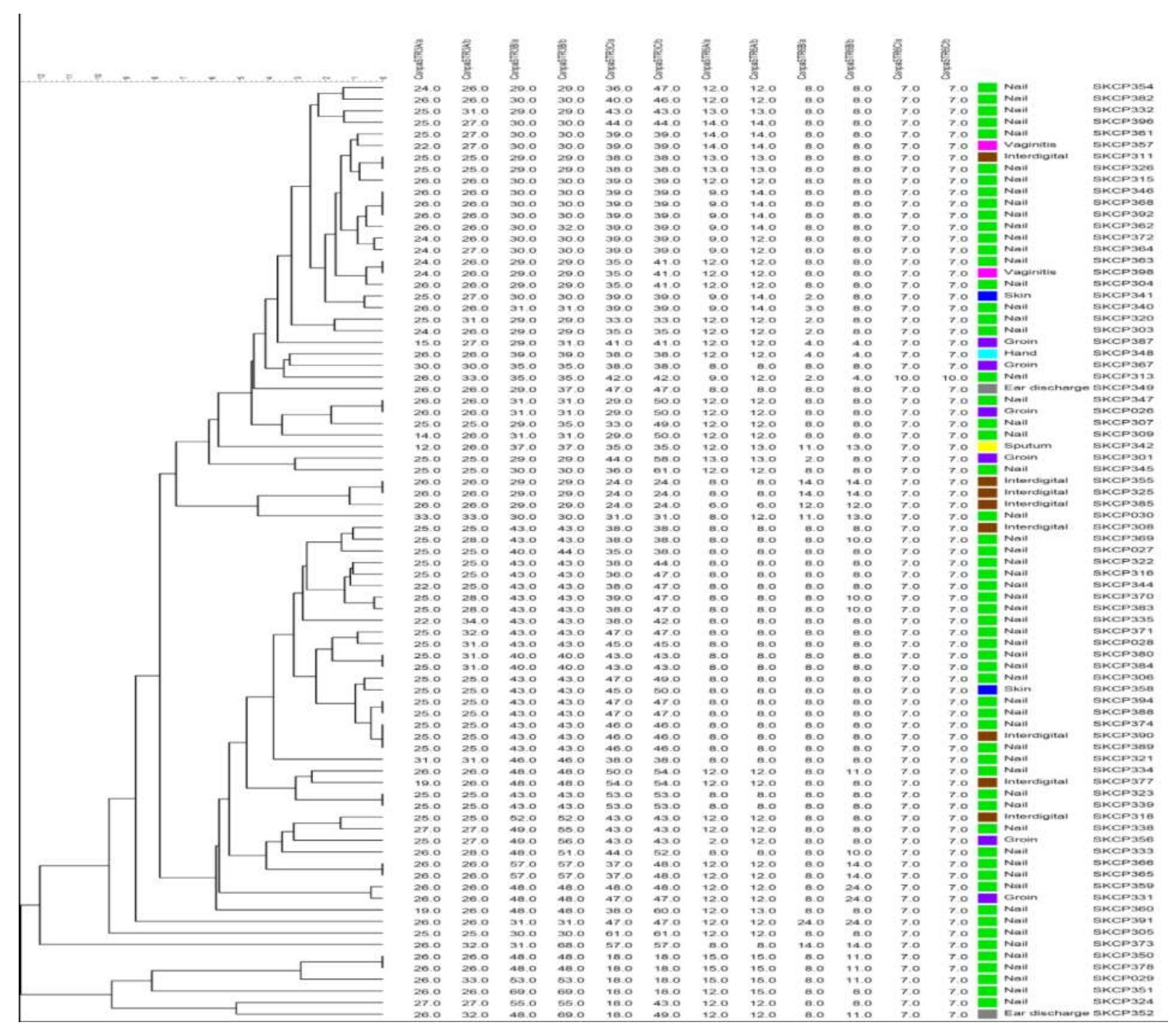

Dendrogram showing genotypic diversity among 81 clinical $C$. parapsilosis isolates obtained from Iran. The scale bar indicates the percentage similarity between the genotypes. The columns after the short tandem repeat patterns represent the source of isolates and isolate number, respectively.

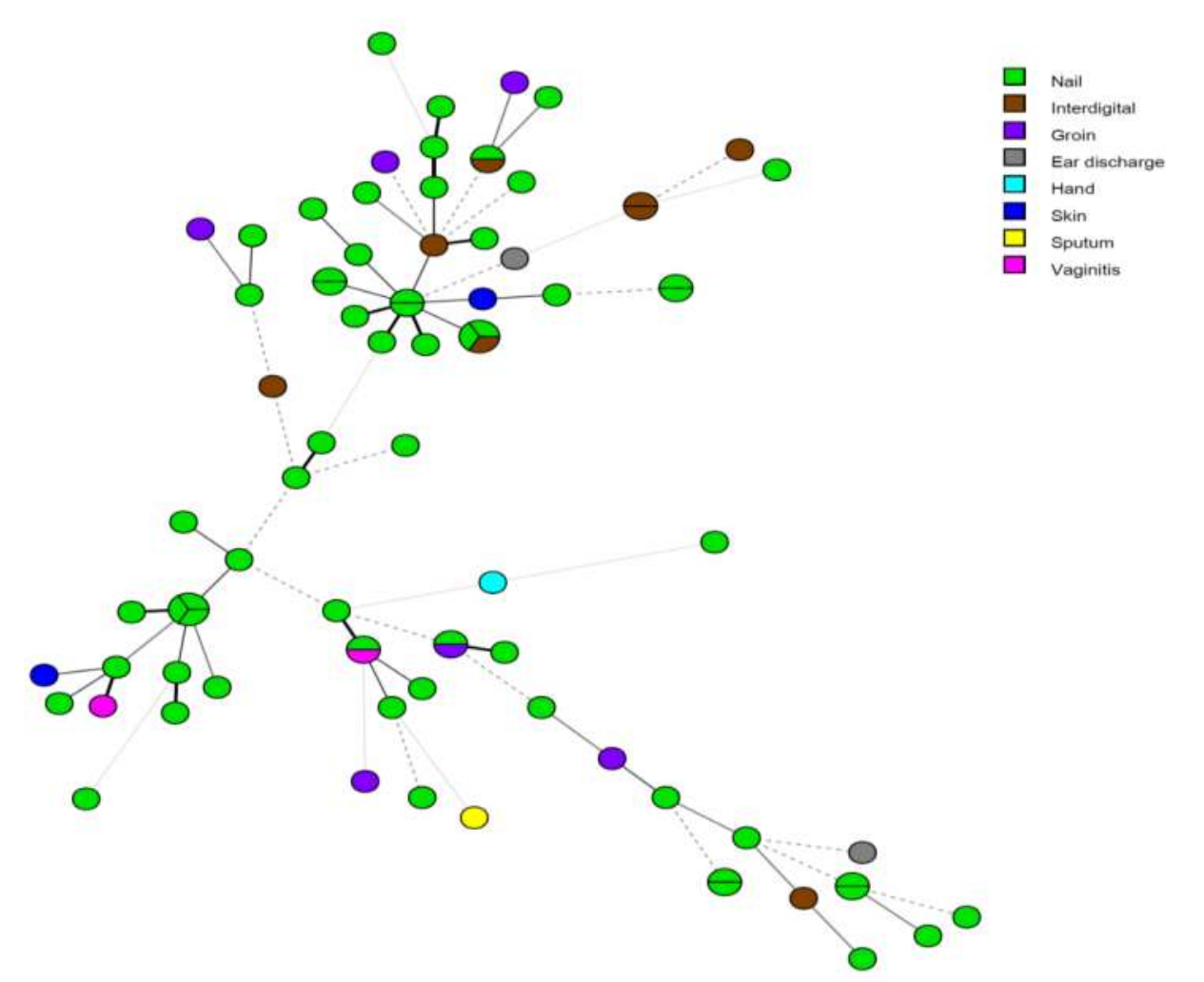

Minimum spanning tree showing the diversity between the genotypes based on sample type categorized analysis. 